\title{
Unrecognised psychopathology in patients with difficult asthma: major mental and personality disorders
}

Lonneke C.J. Prins, Maarten J.M. van Son, Anton R.J. van Keimpema, Jan-Willem G. Meijer, Martina E.F. Bühring and Victor J.M. Pop

\section{Background}

Difficult asthma is a severe subgroup of asthma in which the main feature is uncontrollability of symptoms. Psychopathology is suggested to be prominent in patients with difficult asthma and considered important in its treatment; however, the evidence is scarce.

\section{Aims}

To describe psychopathology in difficult asthma, both major mental and personality disorders, based on diagnostic interviews.

\section{Method}

This study was conducted in a specialised asthma care centre. A total of 51 patients with difficult asthma were diagnosed at the start of the treatment programme using two structured clinical interviews for both major mental (SCID-I) and personality disorders (SCID-II) according to DSM-IV-TR.

\section{Results}

About 55\% of the patients with difficult asthma had a psychiatric disorder of which $89 \%$ was undiagnosed and untreated before being interviewed. About $49 \%$ had a minimum of one major mental disorder of which the cluster of anxiety disorders was the most common cluster of major mental disorders, followed by somatoform disorders. About 20\% were diagnosed with a personality disorder. Of the 10 patients with a personality disorder, 9 had an obsessive-compulsive personality disorder.

\section{Conclusions}

This study demonstrates that more than half of patients with difficult asthma had a psychiatric disorder of which $89 \%$ was unrecognised. This study highlights the importance of offering patients with difficult asthma a psychiatric diagnostic interview and/or a psychiatric consultation as part of their routine medical examination and provision of appropriate psychiatric treatment. Moreover, it highlights the urgency of further research into the role of psychopathology in the development of difficult asthma.

\section{Declaration of interest}

None.

\section{Copyright and usage}

(c) The Royal College of Psychiatrists 2015. This is an open access article distributed under the terms of the Creative Commons Non-Commercial, No Derivatives (CC BY-NC-ND) licence.
Asthma is a lifelong respiratory disease characterised by airway obstruction, airway inflammation and bronchial hyperresponsiveness. ${ }^{1}$ Asthma is a global health problem, ${ }^{1}$ which affects about 1 in 12 people in the USA ( $8 \%$ of 316 million, 25 million) and numbers are increasing every year. ${ }^{2}$ International guidelines on the treatment of asthma advocate a stepwise approach (Global Initiative for Asthma, GINA). ${ }^{1}$ This stepwise approach consists mainly of five steps of pharmacological treatment, the final step including systemic corticosteroids (apart from general recommendations of avoiding asthma triggers, adequate drug compliance, etc.). In most asthmatic patients, the occurrence of symptoms and/or asthma attacks can be controlled with the GINA approach. However, there is a subcategory of asthmatic patients who cannot control their symptoms despite treatment at step 4 or 5 of the GINA guidelines. ${ }^{1}$ In clinical practice, these patients are defined as those who present with difficult asthma, ${ }^{3}$ affecting approximately $5 \%$ of the asthmatic population. ${ }^{4}$ This would mean that about 1.25 million people in the USA and 2.5 million in Europe suffer from difficult asthma. ${ }^{1}$

Difficult asthma has a profound impact on health status and quality of life. ${ }^{5}$ Patients with difficult asthma have frequent exacerbations that can result in hospitalisations, emergency room visits ${ }^{6,7}$ and days of absence from work or school. ${ }^{8}$ Moreover, patients with difficult asthma have an increased risk of sudden asthma death and adverse effects of high-dose corticosteroids. ${ }^{6}$ Although the interest in difficult asthma has grown considerably, the aetiology of difficult asthma is poorly understood. It is well known that major mental disorders are highly prevalent $(31 \%)$ in asthma patients in general ${ }^{9}$ compared with the general population (26\%). ${ }^{10}$ This $31 \%$, however, encompassed both patients with treatable asthma and difficult asthma, which leaves the question whether the higher prevalence could be attributed to patients with asthma in general or to the difficult asthma population specifically. The prevalence of major mental disorders in difficult asthma appears to be high, but is hardly studied. ${ }^{11}$ Two studies by Heaney et al ${ }^{11,12}$ showed a prevalence of $49 \%$ in difficult asthma. However, these studies did not use structured interviews and used ICD-10 criteria instead of the DSM-IV-TR ${ }^{13}$ criteria for assessing mental and personality disorders. These studies did report $81.3 \%$ of mental disorders to be unrecognised. ${ }^{11}$ Although there is a recent publication on personality traits in difficult asthma, ${ }^{14}$ to the best of our knowledge the prevalence of personality disorders in difficult asthma has not been previously reported utilising systematic research diagnostic interviews.

Therefore, the current study assessed the prevalence of psychopathology in difficult asthma focusing on both major mental and personality disorders using research diagnostic interviews according to the DSM-IV-TR. ${ }^{13}$

\section{Method}

\section{Setting}

This study was conducted in Asthma Centre Heideheuvel, Hilversum, The Netherlands, a specialised asthma care centre 
that offers in-patient pulmonary rehabilitation. Asthmatic patients referred to in-patient rehabilitation have highly impaired health status and no satisfactory response to prior medical and nonmedical treatment by asthma specialists, often including outpatient rehabilitation. ${ }^{15,16}$ These complex patients with difficult asthma receive a comprehensive 3 -month rehabilitation programme on an in-patient basis by an interdisciplinary team of healthcare professionals, including pulmonary physicians and specialised healthcare psychologists, as described in detail elsewhere. ${ }^{15,16}$ Patients enter the rehabilitation centre only after a multidisciplinary assessment of four days, confirming their indication. Patients have to be abstinent from smoking.

\section{Participants}

During a period of 17 months, 65 patients with difficult asthma entered the treatment programme and were invited to participate in the current study. This included, apart from the standard intake programme, a psychiatric interview. Seven patients did not give informed consent and another seven patients dropped out during the intake of the rehabilitation programme before diagnostic interviews were performed. The remaining 51 patients consented to participate. Their characteristics are shown in Table 1.

\section{Measurements}

In the first weeks after the start of the programme, each patient was interviewed by trained psychologists with the structured clinical interview (SCID-I) ${ }^{17}$ diagnosing major mental disorders and the structured clinical interview (SCID-II) ${ }^{18}$ diagnosing personality disorders, both according to DSM-IV-TR. ${ }^{13}$

\section{Ethical principles}

Both the institutional medical ethics committee of the Asthma Centre and the medical ethics committee of the Utrecht Medical Centre approved the study protocol according to Dutch law. Patients were sent information about the study before they agreed on participation. All patients gave written informed consent.

\section{Statistical methods}

Statistical analysis was performed using SPSS Statistics for Windows Version 19.0. To determine characteristics and psychopathology of the participants, descriptive statistics were used. Associations between subgroups of patients were analysed using chi-square statistics.

\section{Results}

The characteristics of the 51 patients with difficult asthma are shown in Table 1 . Of these, 41 patients $(80.4 \%)$ were female, $45.1 \%$ were obese, $47.1 \%$ had a medium educational level and $84.3 \%$ middle-class SES. Of the 51 patients with difficult asthma, 28 patients $(54.9 \%)$ had one or more major mental and/or personality disorders (Table 2). Females were more likely to have a major mental disorder (58.5\%) compared with male patients $\left(10.0 \% ; \chi^{2}=7.58\right.$, d.f. $\left.=1, P=0.006\right)$, which was not the case for personality disorders. As can be seen, 25 out of 51 patients (49.0\%) had a major mental disorder, and 10 out of 51 patients (19.6\%) had a personality disorder. Seven out of 51 patients (13.7\%) reported both a major mental disorder and a personality disorder. Ten out of 51 patients (19.6\%) reported more than one major mental disorder and 3 out of 51 patients (5.9\%) reported more than one personality disorder. Overall, anxiety disorders (29.4\%) were the most common cluster of disorders, followed by somatoform disorders (23.5\%) and mood disorders (23.5\%). Airway obstruction (FEV1\%) did not

\begin{tabular}{|lcc|}
\hline Table 1 & Characteristics of patients with difficult asthma ( $n=51)$ \\
\hline Characteristics & $n$ & Means (s.d.) \\
\hline Gender, M/F & $10 / 41$ & 43 (15) \\
\hline Age, years & & \\
\hline Education & 15 \\
Low & 24 \\
Middle & 12 \\
High & \\
\hline SES & 7 \\
Low & 43 \\
Middle & 1 \\
High & \\
\hline BMl & 12 \\
$<24.9$ & 16 \\
$25-29.9$ & 23 \\
$>30$ & \\
\hline FEV1 \% & \\
\hline GINA & 7 \\
Step 4 & 44 \\
Step 5 & \\
SES, socioeconomic status; BMl, body mass index; FEV1 \%, Percentage predicted \\
forced expiratory volume in 1 second; GINA, global initiative for asthma. \\
\hline
\end{tabular}

Table 2 Major mental and personality disorders in 28 out of a sample of 51 patients with difficult asthma who were referred to our specialised asthma care centre (DSM-IV-TR)

\begin{tabular}{|c|c|c|c|}
\hline & $\begin{array}{c}\text { Total } \\
(n=28) \\
n(\%)\end{array}$ & $\begin{array}{c}\text { Females } \\
(n=41) \\
n(\%)\end{array}$ & $\begin{array}{c}\text { Males } \\
(n=10) \\
n(\%)\end{array}$ \\
\hline Psychiatric disorders ${ }^{a}$ & $28(54.9)$ & $26(63.4)$ & $2(20)$ \\
\hline Major mental disorders & $25(49.0)$ & $24(58.5)$ & $1(10)$ \\
\hline Mood disorders & $12(23.5)$ & $12(29.3)$ & $1(10)$ \\
\hline Major depressive disorder & $9(17.6)$ & $9(22.0)$ & 0 \\
\hline Dysthymic disorder & $2(3.9)$ & $2(4.9)$ & 0 \\
\hline Bipolar disorder & $1(2.0)$ & $1(2.4)$ & 0 \\
\hline Anxiety disorders & $15(29.4)$ & $12(29.3)$ & $1(10)$ \\
\hline Social phobia & $4(7.8)$ & $4(9.8)$ & 0 \\
\hline Specific phobia & $5(9.8)$ & $4(9.8)$ & $1(10)$ \\
\hline Post-traumatic stress disorder & $4(7.8)$ & $4(9.8)$ & 0 \\
\hline Panic disorder & $1(2.0)$ & $1(2.4)$ & 0 \\
\hline Obsessive-compulsive disorder & $1(2.0)$ & $1(2.4)$ & 0 \\
\hline Substance disorders & $1(2.0)$ & $1(2.4)$ & 0 \\
\hline Substance abuse disorder & $1(2.0)$ & $1(2.4)$ & 0 \\
\hline Somatoform disorders & $12(23.5)$ & $11(26.8)$ & $1(10)$ \\
\hline Somatisation disorder & $5(9.8)$ & $5(12.2)$ & 0 \\
\hline Undifferentiated somatoform disorder & $4(7.8)$ & $3(7.3)$ & $1(10)$ \\
\hline Pain disorder & $3(5.9)$ & $3(7.3)$ & 0 \\
\hline Personality disorders & $10(19.6)$ & $9(22)$ & $1(10)$ \\
\hline Borderline personality disorder & $1(2.0)$ & $1(2.4)$ & 0 \\
\hline Avoidant personality disorder & $3(5.9)$ & $3(7.3)$ & 0 \\
\hline Obsessive-compulsive personality disorder & $9(17.6)$ & $8(19.5)$ & $1(10)$ \\
\hline Dependent personality disorder & $1(2.0)$ & $1(2.4)$ & 0 \\
\hline Personality disorder NOS & $1(2.0)$ & $2(4.9)$ & 0 \\
\hline
\end{tabular}

differ between the patients with and without a psychiatric diagnosis (data not shown).

Of the 28 patients who were diagnosed with a psychiatric disorder, 3 patients had had a psychiatric diagnosis before referral to the rehabilitation centre: one had an obsessive-compulsive personality disorder (receiving psychotherapy), one had a somatisation disorder (receiving psychotherapy) and one patient had a major depressive disorder (using antidepressants). This means that 25 of the $28(89.3 \%)$ patients had a psychiatric diagnosis 
which was not diagnosed before. Of the 23 patients who were not diagnosed with a psychiatric disorder according to the structured interviews, one patient received antidepressants and another six patients received benzodiazepines for sleeping problems (all prescribed by their GP).

\section{Discussion}

This is one of the first studies in patients with difficult asthma assessing psychopathology using the DSM-IV-TR criteria. ${ }^{19,20}$ The strength of the current study is the involvement of structured interviews (SCID-I and SCID-II) in order to get a DSM-IV-TR classification. The current study showed that over half $(54.9 \%)$ of the patients with difficult asthma referred to the specialised asthma care centre had one or more psychiatric disorders ( $89.3 \%$ of which were previously unrecognised). Specifically, $49 \%$ of all patients had a major mental disorder and 19.6\% were diagnosed with a personality disorder. Heaney et $a l^{1,12}$ reported a similar prevalence (49\%) of major mental disorder in patients with difficult asthma of which $81.3 \%$ was unrecognised. However, this study did not use structured interviews and diagnosed according to ICD-10 criteria. This prevalence is higher in comparison to the $31-34 \%$ reported by two studies by Lavoie et al $l^{9,21}$ in asthmatic out-patients, encompassing both 'normal' asthmatic patients and patients with difficult asthma. The prevalence of $49 \%$ of major mental disorder in the current study is also substantially higher in comparison to the prevalence in the general population in the USA $(26.6 \%)^{10}$ and in The Netherlands $(18 \%) .{ }^{22}$ Similarly, the prevalence of personality disorders of $19.6 \%$ in the current study is also substantially higher compared with the general population in the USA $(9 \%)^{23}$ and in The Netherlands $(13.5 \%) .{ }^{24}$

In the current study, the cluster of anxiety disorders was most common (29.4\%) followed by somatoform disorders (23.5\%) and mood disorders (23.5\%), respectively. Specifically, major depressive disorder was the most common major mental disorder (17.6\%) diagnosed and obsessive-compulsive personality disorder (17.6\%) was the most commonly diagnosed personality disorder.

The current study is among the first using research-based standardised instruments based on DSM-IV-TR criteria to assess a wide range of DSM disorders including personality disorders in difficult asthma. Since we studied a group of patients with complex asthma in one specialised asthma care centre, the question remains open whether these findings are generalisable to the total population of patients with difficult asthma. In the current study, all patients did have difficult asthma according to GINA guidelines. However, patients who smoked, patients without sufficient learning ability for the (intensive) rehabilitation programme (e.g. because of intellectual disability, neuropsychological problems, motivational problems) and patients with known psychiatric or medical diseases interfering with this programme were excluded (as part of general policy of the rehabilitation centre) which might have resulted in an underestimation of the prevalence of psychiatric disorders. And the small number of male participants does not warrant a conclusion about differences between male and female patients with difficult asthma.

About $89.3 \%$ of the patients with difficult asthma in the current study who received a psychiatric diagnosis were not diagnosed before. Because of the dramatic manifestation of difficult asthma, psychiatric symptoms like anxiety or panic can be mistaken for asthma symptoms, which might explain the high number of unrecognised psychiatric disorders. Moreover, stigma about psychiatric disorders, for instance, resulting in being afraid that a psychiatric disorder would negatively impact asthma treatment, could cause unwillingness to acknowledge and accept psychiatric symptoms. ${ }^{25}$
Although beyond the scope of the current study, an important question about the direction of the relation is: is psychopathology a contributor or a consequence of difficult asthma? Psychiatric symptoms could worsen asthma symptoms and the dramatic manifestation of asthma symptoms in difficult asthma could probably cause or worsen psychiatric symptoms. However, the impact of psychiatric symptoms on difficult asthma does not appear to be a straightforward one. ${ }^{11}$ Moreover, because by definition, most patients with difficult asthma use corticosteroids and it is well known that these drugs have major psychotropic side-effects, a possible independent effect of this medication on major mental disorders should be taken into account. ${ }^{26}$ Psychological distress is heightened in patients with severe prednisonedependent asthma ${ }^{14}$ and the use of oral corticosteroids are related to lower quality of life. ${ }^{27}$ Several psychiatric disorders are significantly associated with adult-onset asthma. ${ }^{28}$ Prospective research is needed in which patients who develop difficult asthma after a prior diagnosis of asthma are followed and for patients with a first diagnosis of a psychiatric disorder and with a first diagnosis of asthma, to elucidate on a possible contributing role of psychiatric disorder in difficult asthma.

Both major mental disorders and personality disorders ${ }^{29}$ have a substantial impact on quality of life, which is known to be poor in patients with difficult asthma. ${ }^{30}$ Based on the effect of psychiatric therapy in general, ${ }^{31}$ it is reasonable to suggest that the treatment of major mental and personality disorders also improves quality of life in difficult asthma. In chronic obstructive pulmonary disease (COPD), a severe pulmonary disease in which also high doses of corticosteroids are used, comparable figures of psychiatric disorder are reported. ${ }^{32}$ In this population, the risk of missing diagnoses and treatment of concurrent psychiatric disorder is also high. ${ }^{32}$ Studies on the treatment of psychiatric disorder in COPD show improvement for both physical and psychiatric complaints. ${ }^{32}$ Given these results, one might hypothesise that similar outcomes of treatment might be obtained in a difficult asthma population. Future research is needed to determine the benefit of psychotherapy and/or pharmacotherapy in standard difficult asthma treatment.

This study advocates the importance of offering patients with difficult asthma a psychiatric diagnostic interview and/or a psychiatric consultation as part of their general medical examination since $54.9 \%$ of patients with difficult asthma were diagnosed with a psychiatric disorder, most of them unrecognised and untreated. Furthermore, the results stress the urgency of further research into the potential roles of mental disorders in difficult asthma and of difficult asthma in mental disorders.

\footnotetext{
Lonneke C.J. Prins, MSc, Department of Medical Health Psychology, University of Tilburg, The Netherlands; Maarten J.M. van Son, PhD, Department of Clinical Psychology, Utrecht University, The Netherlands; Anton R.J. van Keimpema, MD, PhD, Asthma Center Heideheuvel, Hilversum, The Netherlands; Jan-Willem G. Meijer, $\mathrm{MD}, \mathrm{PhD}$, Revant, Pulmonary Rehabilitation Center 'Schoondonck', Breda, The Netherlands; Martina E.F. Bühring, MD, PhD, Altrecht Psychosomatic Medicine, Zeist, The Netherlands; Victor J.M. Pop, MD, PhD, Department of Medical Health Psychology, University of Tilburg, The Netherlands

Correspondence: Lonneke C.J. Prins, Department of Medical and Clinical Psychology, Centre of Research on Psychology in Somatic diseases (CoRPS), Tilburg University, PO Box 90153, 5000 LE Tilburg, The Netherlands. Email: Icjprins@gmail.com

First received 17 Jan 2015, final revision 22 Apr 2015, accepted 22 Apr 2015
}

\section{References}

1 Global Initiative for Asthma (GINA). Global Strategy for Asthma Management and Prevention. 2012. http://www.ginasthma.org/guideline. (Accessed February 2014). 
2 Centers for Disease Control and Prevention (CDC). Asthma in the US growing every year. 2011. http://www.cdc.gov/VitalSigns/Asthma/index.html (Accessed February 2014)

3 Chung KF, Wenzel SE, Brozek JP, Bush A, Castro M, Sterk PJ, et al. International ERS/ATS guidelines on definition, evaluation and treatment of severe asthma. Eur Respir J 2014; 43: 343-73.

4 Barnes PJ, Woolcock AJ. Difficult Asthma. Eur Respir J 1998; 12: 1209-18.

5 Chipps BE, Zeiger RS, Borish L, Wenzel SE, Yegin A, Hayden ML, et al. Key findings and clinical implications from The Epidemiology and Natural History of Asthma: Outcomes and Treatment Regimens (TENOR) study. J Allergy Clin Immunol 2012; 130: $332-42$

6 Chung KF, Godard P, Adelroth E, Ayres J, Barnes P, Bel E, et al. Difficult/therapyresistant asthma: the need for an integrated approach to define clinical phenotypes, evaluate risk factors, understand pathophysiology and find nove therapies: ERS Task Force on Difficult/Therapy-Resistant Asthma. Eur Respir $J$ 1999; 13: 1198-208

7 American Thoracic Society. Proceedings of the ATS workshop on refractory asthma: current understanding, recommendations, and unanswered questions. Am J Respir Crit Care Med 2000; 162: 2341-51.

8 Gendo K, Lodewick MJ. Asthma economics: focusing on therapies that improve costly outcomes. Curr Opin Pulm Med 2005; 11: 43-50.

9 Lavoie KL, Bacon SL, Barone S, Cartier A, Ditto B, Labreque M. What is worse for asthma control and quality of life: depressive disorder, anxiety disorder, or both? Chest 2006; 130: 1039-47.

10 Kessler RC, Chiu WT, Demler O, Walters EE. Prevalence, severity, and comorbidity of twelve-month DSM-IV disorders in the National Comorbidity Survey Replication (NCS-R). Arch Gen Psychiatry 2005; 62: 617-27.

11 Heaney LG, Conway E, Kelly C, Gamble J. Prevalence of psychiatric morbidity in a difficult asthma population: relationship to asthma outcome. Respir Med 2005; 99 : 1152-9.

12 Heaney LG, Conway E, Kelly C, Johnston BT, English C, Stevenson M, et al Predictors of therapy resistant asthma: outcome of a systematic evaluation protocol. Thorax 2003; 58: 561-6.

13 American Psychiatric Association. Diagnostic and Statistical Manual of Mental Disorders. (4th edn, text revision). American Psychiatric Association, 2000.

14 Amelink M, Hashimoto S, Spinhoven P, Pasma HR, Sterk PJ, Bel EH, et al. Anxiety, depression and personality traits in severe, prednisone-dependent asthma. Respir Med 2014; 108: 438-44.

15 Van Ranst $D$, Otten $H$, Meijer JW, Van 't Hul A. Outcome of pulmonary rehabilitation in COPD patients with severely impaired health status. Int J Chron Obstruct Pulmon Dis 2011; 6: 647-57.

16 Rijssenbeek-Nouwens LH, Fieten KB, Bron AO, Hashimoto S, Bel EH, Weersink EJ. High-altitude treatment in atopic and nonatopic patients with severe asthma. Eur Respir J 2012; 40: 1374-80.

17 First MB, Gibbon M, Spitzer RL, Williams JBW. User's Guide for the Structured Clinical Interview for the DSM-IV Axis I disorders-Research Version. Biometrics Research Department, New York State Psychiatric Institute, 1996.

18 First MB, Gibbon M, Spitzer RL, Williams JBW, Benjamin LS. Structured Clinical Interview for DSM-IV Axis II Personality Disorders (SCID-II). American Psychiatric Press, 1997.

19 Ten Brinke A, Ouwerkerk ME, Zwinderman AH, Spinhoven P, Bel EH. Psychopathology in patients with severe asthma is associated with increased health care utilization. Am J Respir Crit Care Med 2001; 163: 1093-6.

20 Ten Brinke A, Ouwerkerk ME, Bel EH, Spinhoven P. Similar psychological characteristics in mild and severe asthma. J Psychosom Res 2001; 50: 7-10.

21 Lavoie KL, Cartier A, Labrecque M, Bacon SL, Lemière C, Malo JL, et al. Are psychiatric disorders associated with worse asthma control and quality of life in asthma patients? Respir Med 2005; 99: 1249-57.

22 deGraaf R, ten Have M, Van Dorsselaer S. De Psychische Gezondheid van de Nederlandse Bevolking. NEMESIS-2: Opzet en Eerste Resultaten. Trimbosinstituut, 2010.

23 Lenzenweger MF, Lane MC, Loranger AW, Kessler RC. DSM-IV personality disorders in the National Comorbidity Survey Replication. Biol Psych 2007; 62: 553-64.

24 de Jong A, van der Brink W, Ormel J (eds). Handbook Psychiatrische Epidemiologie. Elsevier / De Tijdstroom, 1999.

25 Jorm AF. Mental health literacy: public knowledge and beliefs about mental disorders. Br J PSychiatry 2000; 177: 396-401.

26 Warrington TP, Bostwick JM. Psychiatric adverse effects of corticosteroids. Mayo Clin Proc 2006; 81: $1361 \mathrm{e}$.

27 Hyland ME, Whalley B, Jones RC, Masoli M. A qualitative study of the impact of severe asthma and its treatment showing that treatment burden is neglected in existing asthma assessment scales. Qual Life Res 2015; 24: 631-9.

28 Alonso J, de Jonge P, Lim CC, Aguilar-Gaxiola S, Bruffaerts R, Caldas-de-Almeida $\mathrm{JM}$, et al. Association between mental disorders and subsequent adult onset asthma. J PSychiatr Res 2014; 59: 179-88.

29 Spitzer RL, Kroenke K, Linzer M, Hahn SR, Williams JB, deGruy FV III, et al. Healthrelated quality of life in primary care patients with mental disorders: results from the PRIME-MD 1000 study. JAMA 1995; 274: 1511-7.

30 Heaney LG, Menzies-Gow A. Difficult Asthma. Jaypee Brothers, 2013

31 Wampold BE. The Great Psychotherapy Debate: Models, Methods, and Findings. Lawrence Erlbaum Associates Publishers, 2001

32 Mikkelsen RL, Middelboe $\mathrm{T}$, Pisinger $\mathrm{C}$, Stage $\mathrm{KB}$. Anxiety and depression in patients with chronic obstructive pulmonary disease (COPD). A review. Nordic J Psychiatry 2004; 58: 65-70. 